\title{
PRAGMATISMO Y GRAMATICALIZACIÓN EN EL CAMBIO LINGÜÍSTICO: SER Y 'ESTAR EN EXPRESIONES DE EDAD
}

\section{INTRODUCCIÓN ${ }^{*}$}

En este artículo discutimos el surgimiento de una nueva categorización como parte de un cambio lingüístico en curso, a saber, el reemplazo de ser por estar en por lo menos dos variantes del español de América. Este reemplazo tiene que considerarse en el marco de un movimiento paulatino en los contextos de ser y estar en general hacia este verbo en la historia del español (Pountain 1982). En nuestra investigación dimos con un grupo específico de contextos donde estar como verbo copulativo con atributos que indican edad parece reemplazar a ser en el español de México y Venezuela (véase también De Jonge 1987). Además, en este lugar proponemos concentrarnos en una pregunta teórica básica: ¿cómo se efectúa la reinterpretación, eslabón imprescindible en todo cambio lingüístico (Leumann 1927)?

\section{Algunos datos esenciales}

En México y Venezuela el uso de estar como verbo copulativo en expresiones de edad está cada vez más generalizado, donde el español peninsular únicamente admite el uso de $s e r^{1}$. Las construcciones en juego son del tipo

* Este artículo es una versión modificada, ampliada y completada de uno de los capítulos de De Jonge 1990a, 1990b y 1991b. Agradezco mucho los comentarios críticos a versiones anteriores de este artículo que me hicieron Reineke Bok-Bennema, Johan Buijsman, Concepción Company, Érica García, Hub Hermans, Dorien Nieuwenhuijsen y Co Vet.

${ }^{1}$ Que yo sepa a través de informantes, este uso innovador de estar en 
i) Juan es joven/viejo (uso peninsular).

En el español de México y Venezuela, además de este tipo, bajo ciertas circunstancias se observan construcciones del tipo

ii) Juan está joven/viejo

cuyo significado no es como el uso permitido de estar en la Península de 'Juan parece más joven/viejo de lo que es', sino que se tendría que interpretar como sinónimo de (i), o sea que (i) y (ii) estarian "saying the same thing in different ways" en el sentido de García².

Para medir el grado de introducción de estar en este nuevo contexto se han recogido todos los ejemplos de expresiones de edad, tanto con ser como con estar para distintos grupos de hablantes mexicanos y venezolanos. En la tabla 1 se dan los datos relevantes a la invasión de estar en las expresiones de edad para cuatro corpora: el habla culta de la ciudad de México (MC), el habla popular de la ciudad de México (MP), el habla culta de Caracas, Venezuela (CC) y el habla de los analfabetos caraqueños $(\mathrm{CA})^{3}$. Estos datos forman la base para el análisis en este artículo.

contextos de edad no se ha observado en otros países latinoamericanos de habla hispana. Ni en la variante del español peninsular considerada más parecida al español americano, el andaluz, se han encontrado ejemplos con uso de estar en dichos contextos, tal como resultó de la consulta de la muestra del habla culta de Sevilla, ofrecida en PINEDa 1983: en los 20 ejemplos encontrados en este material se observa el verbo copulativo ser. Sin embargo, en una conversación personal con un señor granadino de unos 60 años, profesor de matemáticas en un colegio, he observado el único uso peninsular conocido por mí hasta ahora. Cuando el señor estaba hablando de su costumbre de ir de vacaciones solo, sin su familia, para practicar el senderismo, me dijo: "Durante un cierto periodo, cuando mis hijos estaban pequeños, no pude ir de vacaciones" (julio de 1992). Tal como se discute en De Jonge 1990a, el hecho de que esta expresión de edad tenga como sujeto los hijos del informante es un factor importante para la primera fase de la introducción de estar en estos contextos. Sin embargo, de este hecho aislado no podemos sacar la conclusión de que también en España se ha introducido el cambio lingüístico que se estudia en este artículo.

${ }^{2}$ García 1985, pp. 199-200.

${ }^{3}$ Los corpora representan dos estratos sociales de ambas ciudades: un nivel socio-cultural alto (MC y CG) y otro bajo (MP y CA). Los datos de los corpora han sido tomados de LOPE BLANCH 1971 y 1976 y Rosenblat 1979, obras que forman parte del Proyecto de estudio coordinado de la norma lingüistica culta de las principales cudades de Iberoamérica y la Península Ibérica. Dado que el material publicado no rindió suficientes ejemplos para un estudio suficientemente 
TABLA 1

Frecuencias absolutas $(N)$ y relativas (\%) de ser vs. estar en expresiones de edadi en cuatro corpora latinoamericanos

\begin{tabular}{lcrr}
\hline & $N$ total & $N$ ser & $N$ estar \\
\hline México Culto & 159 & $102(64 \%)$ & $57(36 \%)$ \\
Caracas Culto & 192 & $90(47 \%)$ & $102(53 \%)$ \\
México Popular & 157 & $55(35 \%)$ & $102(65 \%)$ \\
Caracas Analf. & 48 & $13(27 \%)$ & $35(73 \%)$ \\
\hline
\end{tabular}

Como se ha discutido en De Jonge 1990a, la introducción de estar en el territorio de las expresiones de edad en México y Venezuela tiene que coincidir con una nueva categorización del concepto de edad: donde antes (y todavía en España) se indicaba la edad de una persona en (casi) cualquier situación con un solo verbo, el copulativo ser, ahora se hace con dos: unas veces ser, y otras veces estar. La manera en que manejan los hablantes de los países en cuestión esta nueva categorización y; lo que es más importante, cómo esta categorización puede diferenciar entre distintos grupos de hablantes, no se puede demostrar independientemente: sólo se puede observar y analizar detenidamente el uso de las expresiones de edad, partiendo de la suposición de que una elección dis-

amplio, recurrimos al análisis de una parte del material no publicado en forma de cintas grabadas o transcripciones de las mismas, hechas en el marco del mismo proyecto. Estos materiales, y otros más, fueron consultados en el Centro de Estudios Hispánicos de la Universidad Nacional Autónoma de México (UNAM), en el Centro de Estudios Lingüísticos y Literarios (CELL) de El Colegio de México y en el Instituto de Filología Andrés Bello (IFAB) de la Universidad Central de Venezuela, instituciones a las cuales estoy profundamente agradecido por su gentileza de ofrecerme total libertad en la consulta de sus materiales tan cuidadosamente elaborados. En el último Instituto menćionado también se consultaron unas grabaciones efectuadas para un proyecto descrito en D'InTRono 1982, de las que se sacaron los datos para CA. En los ejemplos individuales citados en el texto se indicará la fuente por medio del número de la página, si se trata de la parte publicada de los corpora, y del número de la cinta o transcripción, si se trata de la parte consultada en las instituciones mencionadas. La consulta de las partes inéditas de los corpora resultó posible gracias a ayudas de la Fundación Neerlandesa para el Fomento de Investigaciones Tropicales (WOTRO) y del Ministerio de Educación y Ciencias de los Países Bajos. 
tinta de forma - ser o estar - refleja que hay distintos significados subyacentes a esas formas, los cuales implicarían visiones distintas de la realidad. Por lo tanto, la ocurrencia tanto de ser como de estar podría verse como una indicación de que una nueva categorización implícita ha tenido lugar en los contextos de edad.

El hecho de que la introducción de estar a expensas de ser tenga lugar precisamente en los contextos de edad no es sorprendente: el concepto de edad no sólo es una indicación para clasificar a personas en grupos, o sea, para categorizarlas ${ }^{4}$, sino también un fenómeno que implica un cambio constante. En este sentido "edad" es altamente compatible con el verbo estar ${ }^{5}$.

En este artículo, intentaremos discutir la distribución de ser y estar en las expresiones de edad en términos del mensaje comunicativo, resultado de la estrategia del hablante. Distinguiremos contextos en los que la visión de edad es claramente compatible con los significados básicos de los verbos ser o estar, o sea, contextos en los que se clasifica al sujeto mediante la expresión de edad (ser), o expresiones de edad donde el cambio con respecto al sujeto es altamente relevante (estar). Denominaremos a estos casos contextos categóricos, los unos de estar, y los otros de ser.

Por otra parte, también habrá expresiones de edad en que la visión no se puede relacionar directa e indiscutiblemente con los significados básicos de uno de los dos verbos copulativos. En estos casos, el uso de los verbos copulativos puede fluctuar dentro de un mismo grupo de hablantes sin que esto signifique un conflicto con la oposición semántica de los verbos copulativos. Téngase en cuenta, sin embargo, que aquí no se alude a la variación libre, sino más bien a una diferencia individual entre los hablantes en la estrategia para el uso de los verbos, sin consecuencias

${ }^{4}$ Este aspecto hace que la edad sea compatible con el verbo copulativo ser.

${ }^{5}$ Hay que observar, sin embargo, que este cambio es irreversible, al contrario de los "cambios" generalmente indicados mediante el copulativo estar: ésta sería otra justificación del uso peninsular de ser en los contextos de edad. Además, para muchos hispanohablantes (españoles, argentinos etc., que no usan estar en estos contextos) estar se podría clasificar como verbo "estativo", lo cual se podría sentir conflictivo con edad como un proceso continuo. También esta suposición tal vez explique la insistencia de muchos hispanohablantes en el uso de ser, por extraño que resulte este uso para muchos estudiantes de español de habla neerlandesa. 
dañinas para la efectividad de la comunicación. Este tipo de contextos se calificarán de aquí en adelante de neutrales.

El hecho de que se pueda distinguir entre casos claros para ser y estar, por un lado, y casos no-claros - los neutrales - por otro, es de una importancia fundamental para el cambio lingüístico. Son los casos claros de estar, en este caso particular, los que hacen posible la introducción de estar donde antes no aparecía; los casos no-claros hacen posible la expansión de estar en el nuevo contexto en general.

Resumiendo, podría verse el proceso del cambio lingüístico bajo estudio esquemáticamente de la siguiente manera:

i) ser es el único verbo copulativo que se usa en las expresiones de edad, fenómeno en principio compatible con el copulativo estar

ii) las expresiones de edad en que el mensaje es altamente compatible con el significado de estar, los contextos categóricos, hacen posible la introducción de este verbo en el terreno semántico de edad

iii) los contextos neutrales posibilitan la expansión de estar en el terreno semántico de edad sin efectos dañinos para la oposición semántica entre ser y estar.

En primer lugar trataremos de ilustrar la distinción fundamental entre los contextos categóricos y neutrales por medio de un análisis cualitativo de ejemplos individuales sacados del material de investigación, para luego pasar al estudio de las tendencias generales. Para diferenciar entre los significados básicos de ser y estar, partimos de la siguiente distinción, muy inspirada en Falk 19796:

a) ser indica que el predicado nominal tiene que interpretarse como una categorización del sujeto, como en "Juan es gordo", para identificarlo en contraste con otro tipo de personas, como las esbeltas o delgadas

${ }^{6}$ Falk 1979, que habla únicamente de los adjetivos que indican corpulencia y belleza, dice que el complemento nominal indica la norma general del sujeto si aquél se atribuye mediante ser; en cambio, el complemento indica una desviación (cambio) de la norma del sujeto si se atribuye mediante el verbo copulativo estar. Ahora bien, esa desviación es el resultado de ciertas circunstancias en la situación del sujeto. Este enfoque en las circunstancias relevantes a la desviación significa una modifcación que, a mi modo de ver, hace la distinción de Falk más generalmente aplicable que sólo a los adjetivos de corpulencia y belleza. 
b) estar indica la relevancia de las circunstancias en la atribución del predicado nominal al sujeto, como en "Juan está gordo", siendo esto, por ejemplo, el resultado de una depresión de Juan por la cual ha comido mucho últimamente.

\section{LOS CONTEXTOS PROTOTÍPICOS PARA SER Y ESTAR}

En (1) se da un ejemplo de un contexto que contiene elementos que se relacionan fácilmente con el significado de estar: hay una circunstancia en el contexto que hace relevante el cambio (futuro) en la vida del sujeto gramatical. Probablemente sea este tipo de ejemplo el contexto prototípico para la entrada de estar en los contextos de edad. Este ejemplo lo consideramos, pues, un ejemplo categórico para el uso de estar:

(1) $[\mathrm{MC}$, cinta $\mathrm{m}-31$, mujer, 27 años]

...un viaje de uno o dos meses, entonces sería muchísimo tiempo de dejar a mis hijas, que pienso que están muy pequeñas para dejarlas. Tal vez cuando estén un poquito más grandes, me sienta con mayor libertad de actuar y puedo ir disfrutando realmente el paseo...

En (1) no se da una norma (Falk 1979) acerca de la edad de las hijas de alguien, categorizándolas de esta manera entre los distintos tipos de niños según la edad que tienen, sino que es relevante el cambio en la vida futura del sujeto - las hijas- en relación con las circunstancias de este contexto: el hecho de que a la informante le gustaría hacer un viaje, está en conflicto con la situación actual de sus hijas, siendo ellas demasiado pequeñas para sobrevivir sin el cuidado de su madre. Esto sólo será posible cuando sean más grandes: su crecimiento conlleva el tipo de cambio que hace probable esta expectativa. El uso de estar, por lo tanto, es muy apropiado y comprensible en esta situación.

En (2) se da otro ejemplo de un uso comprensible de estar, aunque no corriente en la norma establecida del castellano. Este ejemplo trata la carrera deportiva del informante:

(2) [MC, cinta m-75, hombre, 25 años]

Siento que engordo, siento que... todo, me siento mal, pierdo condición [risas] ... y eso que fumo bastante, o...

Ene. - ¿Entonces no te quieres prepararte [sic] para las olimpiadas? Inf. - No. [Risas] Y además, yo estoy muy viejo para eso. 
Efectivamente, (2) ilustra lo que se ha afirmado arriba en cuanto al significado de estar. Si bien el adjetivo viejo categoriza al hombre como parte del grupo de personas demasiado viejas para practicar el deporte a un nivel profesional, lo que se podía haber hecho mediante el verbo ser, el verbo copulativo estar agrega un aspecto más que relevante, si no esencial en este contexto: el informante indica que las circunstancias relevantes para su estado actual no sólo son el fumar y comer excesivos, sino también su envejecimiento. En términos de Falk 1979, en el momento de hablar claramente se desvía de su norma individual, válida para sí mismo como deportista. En (1) el aspecto relevante era el crecimiento de las niñas, aquí lo es el envejecimiento del informante. Por lo tanto, en ambos casos, el uso de estar se explica básicamente de la misma manera. En vista del cambio que implican, consideramos los contextos en que el adelanto de la edad es, de alguna manera, relevante, como los contextos categóricos para el uso de estar. Estos contextos los denominaremos cronológicos, dada la relevancia del transcurso de los años de la vida.

También ser tiene sus contextos categóricos, o sea, los contextos en los que la visión de edad es compatible con el significado básico de este verbo copulativo. En (3) se discute la edad mínima para las voluntarias en un hospital:

(3) [MC, p. 88, mujer, 55 años]

Ene. - ¿Y hay algún mínimo de edad para entrar, o desde qué edad...?

Inf. - Pues antes era de diez y ocho años. Ahora hemos pensado que pueden ser más chicas.

En este ejemplo, en contraste con (1) y (2), el crecimiento o envejecimiento del sujeto no es relevante. Aquí se trata, en cambio, de una categorización en un grupo con cierta edad, una norma general para la gente que quiere trabajar como voluntario en un hospital. Este valor es compatible con el significado básico de ser: no se está hablando de personas concretas, sino de un grupo imaginable con ciertas características; por lo tanto, los contextos en que la expresión de edad indica la identificación de una persona, son considerados categóricos para el uso de ser. Estos contextos se llamarán de aquí en adelante tipológicos, dado que no indican un aspecto de la vida del sujeto en cuestión, sino el tipo de persona: joven o vieja. 


\section{LOS CONTEXTOS NEUTRALES AL USO DE SER O 'ESTAR}

En los anteriores ejemplos se ha podido relacionar claramente el significado de los verbos copulativos ser y estar con sus contextos, denominados respectivamente tipológios y cronológicos. Hay otros contextos, sin embargo, en que esto no es tan fácil (véase supra, punto iii). Como veremos más adelante, estos contextos, en que el uso de ser o estar es, aparentemente, indiferente, son cruciales en el proceso de cambio lingüístico: aquí los usos de ambos verbos pueden fluctuar sin efectos dañinos para la oposición entre los mismos.

En (4) vemos un ejemplo de este último tipo; el informante cuenta sobre su hijo que alguna vez tuvo la intención de ir a un conservatorio:

(4) [MC, cinta m-83, hombre, 57 años]

... Luis Horacio tuvo una decepción... una decepción muy grande, porque nosotros, cuando estaba niño lo llevamos al Conservatorio Nacional. Y hizo su solicitud y su, su prueba y salió la prueba perfecta. Entonces, fuimos con el director por un. . . lo mandó llamar el director, el maestro Amparán. Y ése nos dijo que Luis Horacio no podía entrar, que porque tenía las manos chicas.

En este ejemplo no hay circunstancias relevantes que expliquen la función de la expresión de edad, tal como sí lo hemos visto en (1) y (2); ni tampoco se está identificando al sujeto: el oyente ya sabe muy bien de quién está hablando el padre. En este ejemplo, lo que se hace es relacionar un momento en el pasado con la edad del sujeto en cuestión, lo cual no identifica al sujeto, sino más bien un momento en su vida, en el pasado. Esta indicación se podría haber dado mediante un número de años (hace dos años $y$ medio) o una expresión temporal (antes, hace mucho tiempo), pero dado que es el sujeto lo que interesa, la edad del sujeto le sirve al hablante como indicación del momento pasado. Su función, por lo tanto, es comparable con la de una expresión temporal. El hecho de que Luis Horacio tuviera las manos chicas no tiene nada que ver con su edad: el director lo rechaza porque sus manos son aparentemente demasiado pequeñas para ser pianista. Esto, por lo tanto, no explica el uso de estar, ni hay motivos para esperar o excluir el uso de ser.

Como hemos indicado arriba, en este contexto no hay elementos que expliquen transparentemente el uso de estar, ni hay 
elementos que lo excluyan o apunten en la dirección de ser. En comparación con éste, en (5) damos un ejemplo parecido, pero con uso de ser:

(5) [MC, cinta Mx-83, hombre, 57 años]

A mi mujer la conocí... la conocí cuando éramos niños.

Ene. - Sí, sí.

Inf. - Sí. . Precisamente, unos años antes de que yo hiciera mi primer concierto en Veracruz.

Tanto como en (4), aquí se está rememorando algo del pasado; la indicación temporal se hace mediante la edad de una persona, en este caso la del hablante y su mujer, lo que luego se especifica con otra indicación temporal. Esto se puede tomar como indicación de que no es el "ser niños" como cualidad lo que es relevante, sino el momento en el pasado. Mientras que en (4) se utilizaba estar, en éste se usa ser sin que podamos explicar cualitativamente esta diferencia de usos de una manera satisfactoria.

El empleo de ser y estar en contextos equivalentes significa que los hablantes tienen una relativa libertad para expresar su visión personal en este tipo de contextos. La función de este tipo de expresiones de edad, distinta a la de los contextos categóricos discutidos arriba, aparentemente admite esta libertad por parte del hablante. Por eso hemos denominado este tipo de casos los contextos neutrales.

Resumiendo podemos decir que en este problema de variación lingüística hay dos tipos de contextos: los categóricos, en los que se puede relacionar con relativa facilidad el mensaje con el significado de los miembros en oposición, y los neutrales, en que no hay indicaciones claras para el uso de uno $\mathrm{u}$ otro miembro. Los categóricos, a su vez, se reparten entre los categóricos para el uso de ser, los cuales hemos calificado de tipológicos, y los categóricos para estar, los cronológicos. En el próximo apartado se explicará cómo la introducción de estar ha tenido lugar a través de los distintos tipos de contextos.

\section{LA INVASIÓN DE ESTAR EN EL TERRITORIO DE EDAD: EL PROCESO DE CAMBIO LINGÜÍSTICO}

En la tabla 1 se ha demostrado que el grado de introducción de estar a expensas de ser no es el mismo en los distintos corpora. En 
vista del análisis cualitativo de los ejemplos, tampoco es de esperar que el grado de introducción sea el mismo en los distintos tipos de contextos: dada la relación semántica entre ciertos contextos y ciertos verbos, es de esperar que en los contextos cronológicos se observe más uso de estar, luego en los neutrales, mientras que en los contextos tipológicos esperamos observar el uso más bajo de estar, o sea, el uso más elevado de ser.

Para controlar esta expectativa, se han clasificado todos los ejemplos de la tabla 1 en los tres tipos de mensaje, y se han medido las proporciones de uso de ser y estar. Los resultados se dan en la tabla 2.

TABLA 2

Frecuencias relativas (\%) de estar (vs. ser) en tres tipos de expresiones de edadi y las frecuencias generales de estar (vs. ser) en los distintos corpora

\begin{tabular}{lrrrr}
\hline $\begin{array}{l}\text { \% estar } \\
\text { (N. tot. })\end{array}$ & Cron. & Neutr. & Tipol. & Total \\
\hline MC & $75 \%(67)$ & $9 \%(34)$ & $7 \%(58)$ & $36 \%(159)$ \\
CC & $90 \%(60)$ & $68 \%(59)$ & $10 \%(73)$ & $53 \%(192)$ \\
MP & $99 \%(72)$ & $61 \%(23)$ & $27 \%(62)$ & $65 \%(157)$ \\
CA & $100 \%(10)$ & $95 \%(19)$ & $37 \%(19)$ & $73 \%(48)$ \\
\hline
\end{tabular}

Efectivamente se observa lo esperado: el porcentaje de estar siempre es más alto en los contextos cronológicos (por ejemplo, CC: $90 \%$ ), menos alto en los neutrales (CC: 68\%), y más bajo en los tipológicos (CC: 10\%). Los resultados no sólo confirman la validez de la distinción entre los distintos tipos de contextos, sino también nuestra presuposición de que hay una relación semántica entre los dos contextos categóricos y los respectivos verbos.

De este hecho sincrónico, se pueden sacar conclusiones diacrónicas (Coseriu 1973): si el porcentaje de estar siempre es más alto en los cronológicos, se desprende que también históricamente estar debe haber comenzado a invadir los contextos cronológicos, para pasar luego a los contextos neutrales y, por último, penetrar en los contextos tipológicos?

${ }^{7}$ Aquí se está sacando una conclusión diacrónica de unos datos sincrónicos. Este punto fue elaborado extensamente en De Jonge 1990a y 1991a. Por 
En relación con estas consideraciones diacrónicas, se plantea el siguiente problema sincrónico (De Jonge 1991b): como hemos visto arriba, desde el punto de vista de sus funciones pueden distinguirse para las expresiones de edad tres tipos discursivos ${ }^{8}$, pero puesto que la lengua dispone de sólo dos verbos, se impone una agrupación funcional de los tres tipos discursivos en dos categorías conceptuales ${ }^{9}$ relacionables respectivamente con los dos verbos ser y estar.

En consecuencia, la extensión del uso de estar a un nuevo tipo discursivo evidentemente no puede darse sin que simultáneamente cambie la agrupación de los tres tipos discursivos en dos categorías, correspondientes a las dos cópulas ${ }^{10}$. Esta reagrupación, a su vez, equivale a una reinterpretación del uso de estar en los contextos de edad y significa un paso crítico en el proceso de cambio.

La pregunta que planteamos ahora es la siguiente: una vez admitido el uso de estar en los contextos de edad, ¿qué es lo que lleva a su reinterpretación? No podemos llegar a comprender dicha reinterpretación sin tomar en cuenta las estrategias que subyacen la elección entre dos alternativas en un contexto dado.

$\mathrm{Al}$ principio de un cambio lingüístico, cuando una forma se usa en un contexto en el que previamente no aparecía, la motivación del hablante para recurrir a esta forma probablemente sea pragmática: son la situación en el mundo real y la necesidad comunicativa las que llevan al hablante a utilizar la forma innovadora ad hoc y de manera no sistemática. En estos casos, la necesidad comunicativa que motiva tal uso de la nueva forma estará reflejada en todo el contexto en general, y no sólo en la presencia de aquélla. De esta manera, en la discusión de los ejemplos (1) y (2), ha sido necesario tomar en cuenta, respectivamente, la situación del hablante que quería viajar y la de las hijas que necesitaban el cuidado de la madre por su corta edad (1), y el pasado deportivo del informante y su condición física en el momento

motivos de espacio lo tomamos como punto de partida para la discusión que sigue.

${ }^{8} \mathrm{La}$ realidad de estas funciones de las expresiones de edad está probada por la evolución "histórica", sugerida en la tabla 2.

${ }^{9}$ LyONS 1968, pp. 429-433 y 270-271.

${ }^{10} \mathrm{Cf}$. ibid., pp. 282-283, donde se discute la recategorización gramatical. 
actual (2). O sea, en los primeros usos (pragmáticos) de la forma nueva estar, seguramente será el contexto más amplio lo que induce al hablante a considerar apropiado dicho uso, y no elementos individuales en el contexto ${ }^{11}$.

Pero entonces surge otra pregunta: ¿la estrategia del hablante permanece invariable a lo largo de un proceso de cambio lingüístico? 'Tal como se desprende de la tabla 2, en MC sólo se observa una introducción limitada del verbo estar en el campo de las expresiones de edad, dado el porcentaje general de $36 \%$. En los demás corpora, estar ya deja de ser la forma numéricamente marcada, puesto que en todos se registran porcentajes de estar mayores del $50 \%$. En esta situación, en que el uso de la forma innovadora se ha generalizado, ¿la estrategia del hablante al elegir entre las dos cópulas sigue siendo la misma?

No es probable que así sea. Al hacerse más frecuente la forma nueva en un contexto dado, automáticamente ocurrirá con más regularidad en determinados contornos sintácticos, reconocibles gracias a su frecuencia de uso. Ahora bien: una asociación "directa" entre forma y rasgos explícitos del contexto es más fácil de establecer que la asociación entre forma y un mensaje global abstracto, expresado en el contexto general. Por ello, es probable que el oyente aproveche tales correlaciones para realizar sus inferencias e identifique el uso de la forma con elementos sintácticos del contexto inmediato, aunque éstos no reflejen directamente la intención comunicativa que haya motivado el uso de la forma ${ }^{12}$. Evidentemente son los rasgos sintácticos del contexto inmediato los que más se prestan a este tipo de asociación.

Esperamos, por lo tanto, que a lo largo de un proceso de cambio, la estrategia del hablante se simplifique cada vez más y que al aumentar el uso de la forma nueva, el contexto relevante para la elección de la cópula se haga cada vez menos extenso y por ende cada vez más fácil y directamente correlacionable con la for-

${ }^{11}$ La clasificación de los tipos de expresiones de edad de la tabla 2 se ha hecho sobre la base del contexto amplio y no (única y necesariamente) de determinados rasgos sintácticos.

${ }^{12}$ Por otra parte, se podría argumentar que el aumento en frecuencia de la forma nueva hace más difícil/compleja la tarea del hablante: si el hablante tiene que considerar con mayor frecuencia un contexto relativamente amplio para determinar cuál de las dos cópulas es la más apropiada, el esfuerzo sería mayor que si tal evaluación se hiciera solamente en contadas ocasiones. Pese al carácter especulativo de esta consideración, creemos oportuno ofrecerla como factor posiblemente relevante. 
ma. En otras palabras: el surgimiento de una forma en un nuevo contexto tendrá una motivación básicamente pragmática, reflejada en el contexto amplio; pero al generalizarse el uso de la forma, la motivación de ese uso se centrará cada vez más en un contexto inmediato fácilmente accesible, o sea, en elementos sintácticos directamente relevantes. Nuestra hipótesis es, pues, que la motivación del uso de una forma nueva se irá haciendo más sintáctica y menos pragmática. Para poner a prueba la hipótesis tendremos que ver, primero, si la supuesta recategorización en el proceso de cambio efectivamente se observa en los corpora investigados, para luego averiguar si la recategorización coincide con un cambio de la estrategia de los hablantes.

Procederemos a investigar nuestra doble hipótesis de la manera siguiente: comenzamos por "categorizar" las expresiones de edad de dos maneras distintas, a fin de ver qué categorización produce una distinción más tajante entre los usos de ser y estar en qué corpora. Nuestro supuesto básico es que la categorización que más diferencie el uso de las formas en alternancia corresponde mejor a la estrategia del hablante ${ }^{13}$. Una vez establecidas las categorizaciones (más) relevantes para los distintos corpora, trataremos de verificar si los corpora que manejan la (re)categorización más avanzada, muestran para la elección de una cópula una mayor relevancia de rasgos contextuales sintácticos en comparación con aquellos corpora para los que vale la categorización menos avanzada.

\section{LA ESTRATEGIA PRAGMÁTIGA Y LA REGATEGORIZAGIÓN}

Como hemos argumentado, la reinterpretación de la motivación para el uso de las cópulas debería reflejarse en una reagrupación de los tipos discursivos de las expresiones de edad ${ }^{14}$. En vista de que las expresiones de tipo "cronológico" son los primeros con-

${ }^{13}$ Este presupuesto se justifica por el siguiente razonamiento: si supiéramos exactamente lo que hacen los hablantes de cada corpus, obtendríamos una distribución perfectamente complementaria entre ser y estar en las dos categorías válidas para el corpus. Como no sabemos exactamente cómo se comportan los hablantes, tratamos de aproximar su estrategia lo mejor posible, y evidentemente será la categorización que más diferencie entre el uso de ser y estar la que más cerca está de lo que en efecto hacen los hablantes del corpus en cuestión.

14 De Jonge 1991b, pp. 500-501. 
textos donde aparece estar, éstos constituirán la primera categoría relevante para el uso de estar. En la próxima etapa, empero, la categoría correspondiente a estar debería incluir también el tipo de contexto semánticamente más parecido al tipo cronológico. Como indicamos en otra ocasión ${ }^{15}$, este tipo más parecido (o semánticamente menos distinto) lo constituyen los contextos neutrales, por ser éste el tipo de contexto "indiferente" al uso de ser y estar; la categoría relevante a ser quedaría limitada exclusivamente a los contextos tipológicos.

El paso crítico en el proceso de cambio, por lo tanto, tendría que ser una reagrupación en las dos categorías de los tipos discursivos, siendo el tipo "clave" que pase de una categoría a otra, el de los contextos neutrales ${ }^{16}$.

En la figura 1 indicamos esquemáticamente las respectivas categorizaciones para las dos etapas del cambio. Suponemos con base en los porcentajes generales de estar que de los corpora bajo investigación, los mexicanos representan diacrónicamente la primera etapa, y los caraqueños la segunda (De Jonge 1991a), en vista de que tanto $\mathrm{MC}$ como MP tienen porcentajes generales de estar más bajos que los corpora caraqueños. Por lo tanto, idealmente México estará más cerca de la categorización conservadora, y Caracas estará más cerca de la avanzada.

\section{Figura 1}

Dos categorizaciones de las expresiones de edad en dos etapas del proceso de cambio Categorización I: etapa temprana (México) Expresiones de edad

\begin{tabular}{|c|c|}
\hline $\begin{array}{l}\text { Categoría estar: contextos } \\
\quad \text { cronológicos }\end{array}$ & $\begin{array}{l}\text { Categoría ser: contextos } \\
\text { neutrales + tipológicos }\end{array}$ \\
\hline \multicolumn{2}{|c|}{$\begin{array}{c}\text { Categorización II: etapa más avanzada (Caracas) } \\
\text { Expresiones de edad. }\end{array}$} \\
\hline $\begin{array}{l}\text { Categoría estar: contextos } \\
\quad \text { cronológicos }+ \text { neutrales }\end{array}$ & $\begin{array}{l}\text { Categoría ser: contextos } \\
\text { tipológicos }\end{array}$ \\
\hline
\end{tabular}

15 Ibid., p. 499.

${ }^{16}$ La agrupación en una categoría de los contextos neutrales y los cronológicos se lograría mediante una extensión del contenido semántico de la categoría relacionada con estar: los contextos cronológicos indican un cambio en el proceso de vida relevante para el mensaje, mientras que los contextos 
Debemos ver ahora si los datos de los respectivos corpora en efecto corresponden a lo esperado, representado en la figura 1. Con tal objetivo hemos clasificado los datos de los cuatro corpora según ambas clasificaciones indicadas. En todos los corpora esperamos, naturalmente, una preferencia de estar en su respectiva categoría, tanto en la categorización I como en la II. Pero si los corpora efectivamente difieren en cuanto a la categorización del tipo discursivo neutral, el grado de polarización entre las dos categorías diferirá según la categorización en juego. O sea, para los corpora más avanzados, Caracas, donde se supone que los contextos neutrales se han incorporado a la categoría de estar, la polarización debería ser mayor según la categorización II. En los corpora menos avanzados en cambio, en los que, todavía, no ha habido una recategorización, la polarización del uso de las dos cópulas debería ser mayor según la categorización I.

TABLA $3 \mathrm{a}$

Distribución estar (vs. ser) en las expresiones de edadide cuatro corpora, categorizadas como a) [cron.] y b) /neutr. + tip.]

\begin{tabular}{|c|c|c|c|c|c|}
\hline$\%$ estar ( $N$ tot. $)$ & a) cron & $\begin{array}{l}\text { tegorización } I \\
\text { b) } \mathrm{n}+\mathrm{t}\end{array}$ & $X^{2}(d f=1)$ & & $\begin{array}{l}\text { odds- } \\
\text { ratio }\end{array}$ \\
\hline $\mathrm{MC}$ & $75 \%(67)$ & $8 \%(92)$ & 75,7 & $(\mathrm{p}<0,001)$ & 37,7 \\
\hline MP & $99 \%(72)$ & $39 \%(85)$ & 62,3 & $(\mathrm{p}<0,001)$ & 111,9 \\
\hline $\mathrm{CC}$ & $90 \%(60)$ & $36 \%(132)$ & 49,0 & $(\mathrm{p}<0,001)$ & 16,3 \\
\hline CA & $100 \%(10)$ & $68 \%(38)$ & 3,1 & $(\mathrm{p}<0,1)$ & - \\
\hline
\end{tabular}

TABLA $3 b$

Distribución estar (vs. ser) en las expresiones de edadide cuatro corpora, categorizadas como a) [cron. + neutr./ y b) [tip.]

\begin{tabular}{lccccc}
\hline \% estar $(N$ tot. $)$ & $\left.a^{\prime}\right) \mathrm{c}+\mathrm{n}$ & $\left.b^{3}\right)$ tip. & $X^{2}(d f=1)$ & $\begin{array}{c}\text { Cotds- } \\
\text { ratio }\end{array}$ \\
\hline MC & $51 \%(101)$ & $7 \%(58)$ & 33,3 & $(\mathrm{p}<0,001)$ & 14,9 \\
MP & $92 \%(95)$ & $27 \%(62)$ & 69,1 & $(\mathrm{p}<0,001)$ & 28,8 \\
GG & $79 \%(119)$ & $10 \%(73)$ & 87,4 & $(\mathrm{p}<0,001)$ & 35,5 \\
GA & $97 \%(29)$ & $37 \%(19)$ & 17,8 & $(\mathrm{p}<0,001)$ & 48,0 \\
\hline
\end{tabular}

neutrales mera y únicamente implican un cambio en el proceso de vida (DE JONGE 1991b, p. 501). 
El objetivo de las tablas $3 a$ y $3 \mathrm{~b}$ es determinar si en efecto se observan tales polarizaciones. En la tabla 3a se indica el grado de polarización del uso de estar en todos los corpora cuando los datos se clasifican según la categorización I; en 3b se muestran las polarizaciones en todos los corpora bajo la categorización II. Hay tres indicaciones del grado de polarización de estar entre (a) y (b) o (a') y (b'):

i) la diferencia de porcentajes de estar entre (a) y (b), o (a') y (b') respectivamente ${ }^{17}$

ii) el coeficiente $\mathrm{X}^{2}$ con su probabilidad correspondiente ${ }^{18}$

iii) el coeficiente odds-ratio ${ }^{19}$.

En las tablas 3 vemos, tal como se concluye en De Jonge (1991b) sobre la base de datos comparables ${ }^{20}$, que en una etapa

${ }^{17}$ En ambas tablas se mostrará en cada columna el porcentaje de estar (sobre el total de estar $+s e r$ ) en la respectiva categoría como dato básico. Por ejemplo, en la columna (a) de la tabla 3a (categorización I) vemos que del total de 67 ejemplos hallados en esta categoría (únicamente contextos cronológicos) el $75 \%$, o sea, 50 ejemplos, tenían estar. De la misma manera están calculados todos los porcentjaes de ambas tablas.

${ }^{18} \mathrm{El}$ coeficiente $\mathrm{X}^{2}$, indicado en la tercera columna, se calcula sobre los números absolutos de las ocurrencias de ser y estar en cada categoría. Por ejemplo, en la tabla $3 \mathrm{a}$, para $\mathrm{MC}$ los números absolutos son: categoría (a) 50 estar y $17 \operatorname{ser}$ (total 67), (b) 7 estar y 85 ser (total 92). Son éstos los números que forman la base para el $\mathrm{X}^{2}$ en la tercera columna. En general, cuanto más alto es el coeficiente, tanto más significativa será la diferencia entre el porcentaje de estar en (a) y (b) o (a') y (b'). La probabilidad $(\mathrm{p}<\ldots$. indica cuán pequeña es la probabilidad de que la diferencia medida se deba al azar. Las probabilidades menores de 0,05 generalmente no cuentan como significativas. Para una descripción de la prueba $\mathrm{X}^{2}$ y sus aplicaciones, véase ButLer 1985, pp. 112-127.

${ }^{19} \mathrm{El}$ odds-ratio es un coeficiente que mide la fuerza de la diferencia en los porcentajes en una comparación (KRUSKaL 1978, pp. 1133-1134). Se calcula sobre la base de los números absolutos de las ocurrencias de ser y estar de la siguiente manera: se multiplican los casos de uso de cada verbo observado en sus respectivas categorías preferidas (o sea, para $\mathrm{MC}$ en la categorización I, tabla $3 \mathrm{a}: 50 \times 85=4250$ ) y se divide este resultado entre el producto de los casos de uso de cada verbo en sus categorías no-preferidas $(17 \times 7=119)$, y el coeficiente obtenido (o sea, 4250:119=35,7) es el odds-ratio. Un odds-ratio puede resultar muy alto debido a un número absoluto muy bajo ( 0 ó 1 ) en una de las celdas de la tabla; tales casos aparecen marcados con ${ }^{*}$, y dichos coeficientes no pueden considerarse como fidedignos.

${ }^{20}$ En De Jonge 1991b la conclusión se basa en datos relacionados con el (no-)uso de adverbios de tiempo, mientras que aquí procede directamente de los tipos discursivos. En las tablas 3, los resultados son aún más netos que los presentados en DE Jonge 1991 b; volveremos al uso de los adverbios de tiempo al discutir la estrategia sintáctica. 
de cambio relativamente temprana, o sea, en México, la categorización I de los contextos de edad arroja una mayor polarización del uso de las cópulas que la categorización II: en MC tanto la diferencia de porcentajes, el $\mathrm{X}^{2}$, así como el ódds-ratio son mayores en I que en II; en MP las diferencias de porcentajes y los $\mathrm{X}^{2}$ son comparables en I y II, pero el odds- ratio es claramente mayor en I: casi 112 en I vs. casi 29 en II. O sea, ambos corpora mexicanos claramente polarizan mejor en la categorización I.

Por el contrario, en los corpora de Caracas, presumiblemente más avanzados en el proceso de cambio (visto el mayor porcentaje general de estar), el uso de estar resulta más polarizado con la categorización II. En CA vemos el efecto de la (re)categorización sobre todo en el $\mathrm{X}^{2}$ y la significación de la diferencia: ésta no es significativa con la categorización $\mathrm{I}(\mathrm{p}<0,1)$, pero sí lo es, en alto grado, con la II $(\mathrm{p}<0,001)$.

Podemos concluir, pues, que para los corpora mexicanos la categorización I establece una diferenciación más nítida entre el uso de ser y estar que la II. Esto quiere decir que los mexicanos usan el verbo estar fundamentalmente en los contextos categóricos, o sea, en cada instancia de uso, ese uso sirve para fines comunicativos directamente relacionables con el contexto mismo. La estrategia del hablante mexicano se puede caracterizar de pragmática, dado que sirve para fines comunicativos en contextos individuales.

En Caracas vemos que hay una diferencia esencial en la categorización del uso de estar en las expresiones de edad con respecto a México. Es de suponer, pues, que también la estrategia de los hablantes caraqueños sea distinta de la de los mexicanos.

\section{LA ESTRATEGIA SINTÁCTICA: GRAMATICALIZACIÓN}

Si México y Caracas representan, tal como suponemos, etapas distintas en el mismo proceso de cambio lingüístico, la diferencia de categorizaciones representa una reinterpretación en ese proceso de cambio. Entonces, la siguiente pregunta es si esta reinterpretación coincide, tal como suponemos, con el paso de una estrategia pragmática a una sintáctica por parte de los hablantes. ¿Cómo podemos averiguar si tal cambio de estrategia efectivamente ha tenido lugar?

Para averiguar esto, en primer lugar se necesitan elementos sintácticos en el contexto inmediato de la cópula que puedan to- 
marse como síntoma del tipo discursivo de las expresiones de edad. Estos elementos sintácticos tienen que ser relacionables con el mensaje general desde dos puntos de vista:

i) los elementos sintácticos tienen que tener una relación semántica con los tipos discursivos

ii) los elementos sintácticos tienen que ocurrir en dichos contextos con gran frecuencia para que el oyente pueda advertirlos e identificarlos con una de las formas en alternancia.

Una vez identificados tales elementos sintácticos, podemos establecer un control parecido al de las tablas 3. Así podremos verificar si

a) la reinterpretación también se observa mediante esta indicación independiente de los tipos discursivos

b) se observa una polarización creciente del uso de estar, al tomar como correlato de la nueva categorización elementos sintácticos más bien que elementos pragmáticos.

Si la reinterpretación efectivamente corresponde a una categorización en términos de correlatos sintácticos, una clasificación de los usos en términos sintácticos dará, para México, una polarización menor que la observada en las tablas 3 (basada en criterios pragmáticos). El motivo es evidente: los elementos sintácticos son únicamente un síntoma (indirecto) de los tipos discursivos -de índole básicamente pragmática. Pero si los rasgos sintáctieos del contexto realmente crecen en importancia durante el cambio e influyen directamente en la elección de formas, un análisis de los datos sobre la base de criterios sintácticos debería representar una mejora -en todo caso nunca un empeoramiento- respecto de los resultados logrados sobre una base pragmática. En resumen: el paso del criterio pragmático al sintáctico se manifestará como una desmejora en la polarización del uso para México (categorización I) y el mantenimiento o mejora en la polarización para Caracas (categorización II).

Debemos comenzar por localizar posibles elementos contextuales fácilmente identificables con los tipos de mensaje que son pragmáticamente relevantes para la elección de la cópula. Creemos haber hallado tal indicación en el uso de los "adverbios" ya o cuando ${ }^{21}$ vs. su ausencia en el contexto, como lo ejemplifican

21 Caracterizamos a cuando como adverbio de tiempo, aunque esto no es lo acostumbrado. Sin embargo, en los casos que manejamos aquí, cuando marca una indicación temporal respecto del verbo. Tal vez por eso Bello llame 
(6) y (7). El ejemplo (6) es muy parecido al ejemplo cronológico (1), pero en (6) observamos, además, un uso del adverbio ya que concuerda muy bien con el contexto, aunque el contexto de por sí podrá haber sido motivo suficiente para explicar el uso de estar. En el ejemplo el informante es enfermera en un hospital:

(6) [MC, cinta m-131, mujer, 55 años]

Ene. - Este, bueno, ¿para ti lo del hospital ha sido un complemento... de tu vida?

Inf. - Sí, fíjate, muy grande. Yo creo que no lo puedo ir a dejar fácilmente... ¡F Fijate que eso no pasa a todas! Porque muchas dicen: ¡Ay no, ya me cansé, ya estoy muy vieja!

En este ejemplo entra en juego el avance de la edad en relación con la vida laboral. En el momento en que uno siente que sobrepasa la norma que relaciona la edad al trabajo, es comprensible que la indique mediante el verbo estar, tal como ocurre en este ejemplo. El (doble) uso de ya subraya la sensación de haber sobrepasado esta norma.

En (7) se da un ejemplo de un contexto neutral, comparable con (4) y (5) en el que también se observa el adverbio cuando en el contexto.

(7) $\left[\mathrm{CC}\right.$, cinta 012, mujer, tercera generación $\left.{ }^{22}\right]$

Aura me dice que cuente de mis viajes. Tendría que empezar por cuando temperaba en Los Teques, en Antímano, en... en Macuto casi no temperaba, no sé porque si . . . sin embargo recuerdo que me... cuando yo estaba chiquita fuimos en Ma. . temperamos una larga temporada en Maiquetía...

A pesar de su ausencia en algunos pocos casos, el uso de cuando es casi inevitable en los contextos neutrales: si el hablante quiere llevar al oyente a un momento en el pasado mediante una indicación de la edad de uno de los protagonistas, lo más efectivo es

a cuando "adverbio relativo de tiempo" (Bello 1958, p. 374, §1239). Centrándonos en su función, hemos considerado que cuando y ya son básicamente adverbios temporales.

22 En el corpus de Caracas se ha dividido la edad de los informantes en tres grupos: primera, segunda y tercera generación, siendo la primera la más joven, entre 25 y 35 años de edad, la segunda entre 36 y 55 , y la tercera de 56 en adelante. 
el uso del adverbio cuando. En las expresiones de edad tipológicas, por el otro lado, el aspecto temporal está ausente por definición, dado que se trata de una caracterización del sujeto, por lo cual no se espera hallar ningún tipo de adverbio temporal en los contextos tipológicos, tal como se ejemplifica en (8), en que una mujer habla de sus experiencias laborales cuando trabajaba de sirvienta en casa de una familia.

(8) $[\mathrm{MP}$, cinta Mhp-2, mujer, 57 años]

Inf: -Era un general muy rico. Es que después me separé, y sé que quedaron arrumados, no sé por qué. Era muy rico. Yo era chica y veía ¡unos bolsones de dinero que entraban! Pero que apenas podía el chofer con las bolsas.

En este ejemplo no hace falta trasponerse al pasado, porque el oyente ya está ahí, como se ve en la frase era un general muy rico. La expresión de edad sirve para enfatizar su actitud al ver las cantidades de dinero que entraban en la casa: el hecho de que quedara muy impresionada se debía en parte a que era muy chica. Por lo tanto, la expresión de edad da una caracterización del tipo de persona a quien le impresiona esta clase de evento, y el uso, tanto de cuando, como de ya, estaría en contradicción con el contexto ${ }^{23}$.

Como señalamos en la sección anterior, la relación entre rasgo sintáctico y tipo discursivo tiene que ser semántica por un lado, y numérica por otro. $\mathrm{Y}$, como acabamos de ver, en efecto es posible establecer una relación semántica entre ya y las expresiones de edad de tipo cronológico, cuando y el contexto neutral, $\mathrm{y}$, finalmente, la ausencia de cualquier tipo de adverbio y el tipológico. En la tabla 4 se investiga si esta relación también es numérica.

Tal como sale de la tabla 4, no es imposible la ocurrencia de ya o cuando en otros que sus contextos icónicos, como lo demuestra también el ejemplo $(9)^{24}$ :

(9) [MP, cinta Mhp-8, hombre, 32 años]

Ene. - ¿Qué le gustaría para sus hijos?

${ }^{23}$ Como se verá más adelante en la tabla 4 y su discusión, el uso de cuando o ya en un contexto tipológico no es absolutamente imposible.

${ }^{24}$ Este ejemplo sirve de ilustración extrema del fenómeno de la ocurrencia de los adverbios de tiempo en otros contextos que los esperados. 
Inf. - Pues, para mis hijos, este, me gustaría que estudiaran lo más que ellos pudieran estudiar, para que se a... se abrieran campo aquí en la vida, que no sufrieran como quizás sufra yo un poquito, que ellos sean lo que más me interesa, útiles a su patria, útiles a sí mismo, y útiles a, a, cuando eh ya sean mayores, útiles a su familia...

\section{TABLA 4}

Frecuencias absolutas y relativas ${ }^{25}$ de ya, cuando y $\emptyset$ en $M C, M P, C C$ y $C A$, divididos en tres tipos de contextos

\begin{tabular}{|c|c|c|c|c|}
\hline \multirow{4}{*}{$\begin{array}{l}\quad \text { (Cron } \\
N_{1}(\%) \\
\mathrm{MC}\end{array}$} & \multirow{2}{*}{$\begin{array}{l}\text { ión cronológica, } \\
\text { Cron. }\end{array}$} & \multirow{2}{*}{$\begin{array}{c}\text { Neutr.: función neutral; } \\
\text { Neutr. }\end{array}$} & \multicolumn{2}{|c|}{ Tip.: función tipológica) ${ }^{26}$} \\
\hline & & & Tip. & Total \\
\hline & & & & \\
\hline & $15(65 \%)$ & $\emptyset(0 \%)$ & $8(35 \%)$ & 23 \\
\hline cuando & $4(13 \%)$ & $20(67 \%)$ & $6(20 \%)$ & 30 \\
\hline$\emptyset$ & $40(49 \%)$ & $2(2 \%)$ & $40(49 \%)$ & 82 \\
\hline MP & & & & \\
\hline ya & $32(70 \%)$ & $\emptyset(0 \%)$ & $14(30 \%)$ & 46 \\
\hline cuando & $3(13,5 \%)$ & $14(63,5 \%)$ & $5(23 \%)$ & 22 \\
\hline$\emptyset$ & $30(41 \%)$ & $7(9 \%)$ & $37(50 \%)$ & 74 \\
\hline $\mathrm{CG}$ & & & & \\
\hline$y a$ & $7(64 \%)$ & $\emptyset(0 \%)$ & $4(36 \%)$ & 11 \\
\hline cuando & $19(30,5 \%)$ & $40(\mathbf{6 4}, \mathbf{5 \%})$ & $3(5 \%)$ & 62 \\
\hline$\emptyset$ & $22(22,5 \%)$ & $13(13 \%)$ & $63(64,5 \%)$ & 98 \\
\hline CA & & & & \\
\hline$y a$ & $3(75 \%)$ & $\emptyset(0 \%)$ & $1(25 \%)$ & 4 \\
\hline cuando & $1(5 \%)$ & $15(71 \%)$ & $5(24 \%)$ & 21 \\
\hline$\emptyset$ & $5(24 \%)$ & $3(14 \%)$ & $13(62 \%)$ & 21 \\
\hline
\end{tabular}

Sin embargo, no forma parte de los ejemplos tabulados en la tabla 4 , por tratarse de un caso tanto de cuando como ya.

${ }^{25}$ Los porcentajes han sido calculados de la siguiente manera: para todas las ocurrencias de cada (no-)adverbio, se indica el porcentaje observado en cada contexto discursivo. Así, de todos los ya en MC (23, que por lo tanto corresponden a $100 \%$ ), 15 aparecen en contextos cronológicos (lo que da un porcentaje de 65$)$, ninguno en contextos neutrales $(0 \%)$ y 8 en contextos tipológicos $(35 \%)$.

${ }^{26}$ Hemos observado más adverbios de tiempo que pueden tener influencia en la elección de la cópula. Sin embargo, los ejemplos eran tan escasos en comparación con ya y cuando que no cumplían con el criterio de la frecuencia suficiente para identificarse con uno de los verbos. Para estos datos y las demás pruebas basadas en las indicaciones contextuales ya, cuando y $\emptyset$, se han tomado los contextos en los cuales aparecen sólo los adverbios mencionados, 
A pesar del uso tanto de cuando como ya, esta expresión de edad no da ni una visión cronológica ni neutral. Como se trata de la capa social baja de la ciudad de México (México Popular), para ser útiles a su familia, estos niños tienen que ser adultos, o por lo menos lo suficientemente grandes como para poder trabajar y ganar dinero. Se trata, pues, de una caracterización futura de los hijos del informante, y por consecuencia, este ejemplo fue clasificado como tipológico.

El hecho de que también ocurran en otros contextos no le quita fuerza a nuestro argumento: para la identificación de los adverbios con sus contextos icónicos hace falta una relación semántica y una numérica. Esta relación numérica no necesariamente tiene que ser absoluta, pero sí observable: la coincidencia tiene que darse con cierta frecuencia para ser observada por el hablante/oyente. Que se observa una relación numérica entre los adverbios y los tipos discursivos sale claramente de la tabla 4 , donde mostramos la correlación para los distintos corpora. Aparece en negritas el mayor porcentaje de uso para cada (no-)adverbio para cada tipo discursivo; éste corresponde siempre al (no-) adverbio esperado desde el punto de vista cualitativo.

En vista de la relación tanto semántica como numérica entre los (no-)adverbios y los tipos discursivos, parece lícito tomar aquéllos como síntomas "rápidos" y directos de éstos. Procedemos ahora a hacer la misma prueba que en las tablas 3 , pero la clasificación de los casos de uso de ser y estar en las categorizaciones I y II de las tablas 3 no se hará sobre la base del tipo discursivo, sino de la presencia/ausencia de los adverbios de tiempo, tal como vienen indicadas en la tabla 4 . O sea, donde las tablas 3 dice "cron.", la tabla que presentaremos adelante dirá "ya";

excluyéndose combinaciones de ya y cuando o uno de estos dos con otros adverbios de tiempo por no poder juzgar cuál de los adverbios prevalecía en la elección del verbo. En la tabla que sigue indicamos los casos excluidos de los cálculos.

\begin{tabular}{lcccl}
\multicolumn{5}{c}{ Casos excluidos de la tabla 4} \\
& $M C$ & $M P$ & $C C$ & $C A$ \\
ya +cuando & 4 & 4 & 5 & $\emptyset$ \\
otro adverbio & 20 & 11 & 16 & 2 \\
total & 24 & 15 & 21 & 2 \\
\% del total tabla $4:$ & $15 \%$ & $10 \%$ & $11 \%$ & $4 \%$
\end{tabular}

En vista de que los casos excluidos representan porcentajes bajos a ínfimos de los respectivos totales, podemos concluir que los ejemplos manejados en la tabla 4 constituyen una muestra representativa de los corpora en su totalidad. 
donde "neutral", "cuando" y donde "tip.", “ $\emptyset$ ", y las agrupaciones se harán sobre la base de estos elementos contextuales.

Puesto que los adverbios de tiempo y su ausencia pueden tomarse como indicación independiente de los tipos discursivos, sería de esperar que se observara el mismo patrón que hemos observado en las tablas 3 , o sea, que los corpora mexicanos polaricen el uso de ser y estar más bajo la categorización I que bajo la II, mientras que los corpora caraqueños lo hagan más bajo la categorización II que bajo la I. En las tablas 3 hemos visto que México y Caracas coincidían en revelar una polarización más pequeña del uso para la categorización no-preferida, vs. una polarización más grande en la preferida. En las tablas 3 vemos además, a través de las diferencias de porcentajes y los distintos coeficientes, que el grado de polarización en los corpora de tamaño comparable (MC, MP y CC) es equiparable en las respectivas categorizaciones favorables. El objetivo de este nuevo control no es, empero, demostrar lo ya establecido en las tablas 3 , sino dar un paso adelante en la identificación de la estrategia de uso.

Ahora bien, si los elementos sintácticos son más relevantes para los hablantes de Caracas que para los de México, debemos esperar una mayor polarización del uso de estar en Caracas bajo la categorización II con criterio sintáctico que en México bajo la categorización I sobre base sintáctica, puesto que en México la categorización (todavía) no puede ser sintáctica: la categorización I no permite una estrategia sintáctica en el uso de estar con la presencia de adverbios de tiempo, dado que los adverbios ya y cuando están en categorías distintas. La categorización II, en cambió, sí permite - pero no fuerza - una estrategia sintáctica, porque en esta categorización los dos adverbios sí están en la misma categoría.

Si el control a presentar fuera únicamente una indicación independiente de la validez de las tablas 3, no tendríamos motivo para esperar una mayor polarización en Caracas: es nuestra hipótesis en cuanto a la progresiva relevancia de los elementos sintácticos en el proceso de cambio lingüístico que lo predice. Esta diferencia entre la polarización en México y Caracas se revelaría, de nuevo, en una mayor diferencia entre los porcentajes de estar, un mayor grado de significación de los resultados, expresado en el coeficiente $\mathrm{X}^{2}$ y su probabilidad $(\mathrm{p}<\ldots)$ y/o coeficientes odd-ratio más altos en Caracas (sobre todo en $C \mathrm{C}$, dada su buena comparabilidad numérica con MC y MP) en la categori- 
zación II sintáctica que los corpora mexicanos en la categorización I sintáctica. En las tablas 5 damos los resultados de las categorizaciones sintácticas (esto es, sobre la base de adverbios de tiempo). La tabla $5 a$ da los totales en que están basados los resultados de $5 b$ y $c$.

\section{TABLA 5a}

Distribución estar (vs. ser) en expresiones de edad con ya, cuando $y \emptyset$

\begin{tabular}{lccc}
\hline$\%$ estar (N tot.) & ya & cuando & $\emptyset$ \\
\hline MC & $65 \%(23)$ & $17 \%(30)$ & $35 \%(82)$ \\
MP & $83 \%(46)$ & $41 \%(22)$ & $62 \%(74)$ \\
GC & $82 \%(11)$ & $68 \%(62)$ & $35 \%(98)$ \\
GA & $75 \%(4)$ & $81 \%(21)$ & $62 \%(21)$ \\
\hline
\end{tabular}

TABLA $5 b$

Distribución estar (vs. ser) en expresiones de edad, categorizadas como a) $[\mathrm{ya}] y$ b) [cuando $+\emptyset]$

\begin{tabular}{lccccc}
\hline & \multicolumn{3}{c}{ Categorización sintáctica $I$} & & odds- \\
$\%$ estar $(N$ tot.) & a) ya & $b) \mathrm{c}+\emptyset$ & $X^{2}(d f=1)$ & ratio \\
\hline MC & $65 \%(23)$ & $30 \%(112)$ & 8,6 & $(\mathrm{p}<0,01)$ & 4,3 \\
MP & $83 \%(46)$ & $57 \%(96)$ & 9,2 & $(\mathrm{p}<0,01)$ & 3,5 \\
CC & $82 \%(11)$ & $48 \%(160)$ & 3,6 & $(\mathrm{p}<0,1)$ & 5,0 \\
CA & $75 \%(4)$ & $71 \%(42)$ & 0,2 & $(\mathrm{p}<0,7)$ & 1,2 \\
\hline
\end{tabular}

TABLA $5 c$

Distribución estar (vs. ser) en expresiones de edad, categorizadas como $\left.a^{\prime}\right)$ (ya + cuando] y b') [ø]

\begin{tabular}{|c|c|c|c|c|c|}
\hline \multirow[b]{2}{*}{ \% estar ( $N$ tot.) } & \multicolumn{3}{|c|}{ Categorización sintáctica II } & & \multirow{2}{*}{$\begin{array}{c}\text { odds- } \\
\text { ratio }\end{array}$} \\
\hline & $\left.a^{\prime}\right)$ уа $+\mathrm{c}$ & $\left.b^{\prime}\right) \emptyset$ & $X^{2}(d f=1)$ & & \\
\hline $\mathrm{MG}$ & $38 \%(53)$ & $35 \%(82)$ & 0,1 & $(p<0,8)$ & 1,1 \\
\hline MP & $69 \%(68)$ & $62 \%(74)$ & 0,5 & $(p<0,5)$ & 1,4 \\
\hline GC & $70 \%(73)$ & $35 \%(98)$ & 20,7 & $(p<0,001)$ & 4,4 \\
\hline CA & $80 \%(25)$ & $62 \%(21)$ & 1,06 & $(p<0,5)$ & 2,5 \\
\hline
\end{tabular}

Las tablas $5 \mathrm{~b}$ y c efectivamente muestran una imagen comparable a la de las tablas 3 . Lo que es más importante es que en 5 a y b las categorizaciones están hechas sobre la base de un crite- 
rio sintáctico, mientras que la categorización de las tablas 3 está hecha sobre la base de una valoración de los contextos individuales, o sea, un criterio pragmático, relacionado con la acción comunicativa individual. Ahora bien, si hemos observado que en las tablas 3 los grados de diferenciación entre los corpora de tamaño comparable bajo la categorización más favorable eran equiparables en $5 \mathrm{~b}$ y c vemos que hay un mayor grado de diferenciación del uso de estar bajo la categorización sintáctica II en Caracas en comparación con México bajo la sintáctica I, sobre todo en el corpus numéricamente más representativo, CC. En la tabla 6 resumimos los datos en cuanto a la significación de los mejores datos en las tablas 3 y 5 para los únicos dos corpora completamente comparables - MC y CC por el estrato social y el número general de ejemplos - para mejor comparación del grado de polarización en las distintas estrategias.

\section{TABLA 6}

Grado de polarización, expresado en el coeficiente $X^{2}$, en $M C$ y $C C$ en sus mejores categorizaciones respectivas según las dos estrategias

\begin{tabular}{lcc}
\hline $\mathrm{X}^{2 / \mathrm{p}}<\ldots$ Estrategia: & Pragmática (tablas 3) & Sintáctica (tablas 5) \\
\hline Corpus/categorización: & & \\
$\mathrm{MC} / \mathrm{I}$ & $75,7 / \mathrm{p}<0,001$ & $8,6 / \mathrm{p}<0,01$ \\
$\mathrm{CC} / \mathrm{II}$ & $87,4 / \mathrm{p}<0,001$ & $20,7 / \mathrm{p}<0,001$ \\
\hline
\end{tabular}

Si los resultados de las tablas 5 fuesen únicamente una prueba independiente de la validez de la prueba pragmática de las tablas 3, no tendríamos motivo para esperar un aumento en la significación de los resultados de $\mathrm{CC}$ con respecto a $\mathrm{MC}$ en la prueba sintáctica (tablas 5). Empero, en las tablas 3 la significación para MC es muy comparable a la de CG. La pregunta es ¿qué quiere decir este aumento de la singnificación de los resultados en $\mathrm{CC}$ en las tablas 5 con respecto a MG?

El aumento de la polarización para CG en las tablas 5 únicamente puede significar que en este corpus la presencia de los adverbios de tiempo en el contexto correlaciona mejor con la elección de cópula que en los corpora mexicanos, lo que sugiere la existencia de una estrategia sintáctica. Entonces, por lo menos en $\mathrm{CG}$, lo que es relevante en la estrategia del hablante ya no sería sobre todo (o sólo) el contexto global, sino cada vez más la pre- 
sencia (o ausencia) de un rasgo sintáctico específico en este contexto, como lo sería la presencia (o ausencia) de ya o cuando.

\section{Conclusión}

En este artículo hemos intentado demostrar que en el proceso de cambio lingüístico no sólo se produce una recategorización del concepto expresado - en este caso la edad - sino que también cambia la estrategia del hablante al elegir entre las dos formas en alternancia. Como hemos visto reflejado en los corpora de México, en las primeras instancias del uso de estar en expresiones de edad, la motivación del hablante es sobre todo pragmática: la elección de forma depende del deseo comunicativo en cada contexto individual. En Caracas hemos observado, sin embargo, una influencia creciente en el contexto de unos elementos sintácticos, los adverbios de tiempo, en la elección de forma.

Este cambio de estrategia encuentra sus orígenes, a nuestro modo de ver, en la interacción entre hablante y oyente. Si bien el hablante usa la forma nueva con cierta intención comunicativa, observable únicamente en el contexto más amplio, es poco probable que el oyente observe todos los reflejos de esa intención por parte del hablante. Al aumentar el uso de estar, aumenta también la posibilidad de que el oyente identifique el uso de esta forma nueva con elementos sintácticos. Cuando el oyente, habiendo observado y (re)interpretado los usos de las formas, asuma su natural condición de hablante, actuará según sus propias observaciones, y no según la intención del hablante inicial, dado que ésta le es fundamentalmente desconocida.

Los resultados que hemos observado sugieren que el proceso de cambio comienza en el marco de una motivación pragmática dirigida hacia el mensaje individual. Pero en el transcurso del cambio lingüístico, cuando la forma nueva se hace cada vez más frecuente, la motivación del uso de una forma u otra se hace cada vez más sintáctica, influyendo cada vez menos el contexto global y cada vez más elementos sintácticos directamente observables. Dado que se trata de elementos directamente observables, esta estrategia lleva en sí los elementos necesarios para convertirse en regla. Por lo tanto, el fenómeno que acabamos de observar significa uno de los primeros pasos hacia la gramaticalización en el proceso de cambio lingüístico: vemos que el uso de estar en las expresiones de edad en México es más "libre” en el sentido de que 
los hablantes lo utilizan para expresar fines comunicativos específicos en contextos individuales. En Caracas, empero, los hablantes son más restringidos en su elección de las cópulas por elementos sintácticos - ya y cuando - dentro del contexto, lo cual implica un grado más alto en la escala de gramaticalización del uso de estari27.

Seguir este rastro posiblemente nos llevará a soluciones para algunos más de los muchos porqués que rodean el proceso de cambio lingüístico.

Bob DE JoNGE

Rijksuniversiteit Groningen

\section{BIBLIOGRAFÍA}

Bello, Andrés, y Rufino J. Cuervo 1958. Gramática de la lengua castellana. Sopena, Buenos Aires.

Butler, Christopher 1985. Statistics in linguistics. Basil Blackwell, New York-Oxford.

Coseriu, Eugenio 1973. Sincronía, diacronía e historia. Gredos, Madrid.

$F_{A L K}, J$. 1979. "Visión de norma general vs. norma individual", St $N_{4}, 51,275-306$.

García, E. C. 1985. "Shifting variation", Lingua, 67, 189-224.

D'Introno, F., y H. Casalta 1982. "Lingúística y alfabetización", en $E l$ español del Caribe. Ed. O. Alba. Universidad Católica Madre y Maestra, Santiago, República Dominicana, pp. 65-86.

DE JONGE, R. 1987. "Estar comes of age", en Linguistics in the Netherlands 1987. Eds. F. Beukema and P. Coopmans. Foris, Dordrecht, pp. 101-110.

— 1990a. Sprekersstrategieën en taalverandering: "ser" en "estar" in leeftijdsuitdrukkingen. Leiden Universiteit [tesis de doctorado].

— 1990b. "Ser y estar y expresiones de edad: cambio lingüístico y reinterpretación", en Actas del IX Congreso ALFAL. (En prensa).

- 1991a. "Verschuivingen in het gebruick van ser en estar in LatijnsAmerika", en Spaans in onderwijs onderzoek en bedrijfsleven 2. Eds. C. van Esch \& M. Steenmeijer. Universidad de Nimega, Holanda, pp. 50-61.

- 1991b. "La cosa (no) es como está", en ACIEA(3). T. 1, pp. 495-504.

KrụsKal, William, and JUdith M TANUR (eds.) 1978. International Encyclopedia of Statistics. T. 2. MacMillan, New York-London.

LehmanN, CH. 1986. "Grammaticalization and linguistic typology", GLing, $26,3-22$.

LeumanN, M. 1927. "Zum Mechanismus des Bedeutungswandels", Indogermanische Forschungen, 45, 105-118. 
Lope Blanch, Juan M. 1971. El habla culta de la ciudad de México: materiales para su estudio. UNAM, México.

1976. El habla popular de la ciudad de México: materiales para su estudio. UNAM, México.

LyONS, JOHN 1968. Introduction to theoretical linguistics. Cambridge University Press, Cambridge.

PINEDA, M. A. DE 1983. Sociolingüistica andaluza. T. 2: Encuestas del habla urbana de Sevilla: nivel culto. Universidad, Sevilla.

Pountain, Ch. J. 1982. "*Essere/stare as a Romance phenomenon", en Studies in the Romance verb. Eds. N. Vincent \& M. Harris. Cremona Vol, London, pp. 139-160.

Rosenblat, Angel 1979. El habla culta de Caracas: materiales para su estudio. Universidad Central de Venezuela, Caracas. 